\title{
Water-Mediated Electronic Structure of Oligopeptides Probed by Their UV Circular Dichroism, Absorption Spectra, and Time-Dependent DFT Calculations
}

\author{
Anshuman Kumar, ${ }^{\dagger}$ Siobhan E. Toal, ${ }^{\ddagger}$ David DiGuiseppi, ${ }^{\Uparrow}$ \\ Reinhard Schweitzer-Stenner, "卂 and Bryan M. Wong ,t
}

†Department of Chemical \& Environmental Engineering, Materials Science \& Engineering Program, Department of Chemistry, and Department of Physics \& Astronomy, University of California-Riverside, Riverside, CA 92521, USA $\ddagger$ Department of Chemistry and Biochemistry, Rowan University, Glassboro, NJ 08028, USA

IDepartment of Chemistry, Drexel University, Philadelphia, PA 19104, USA

Corresponding authors:

R. S.-S.: E-mail: rs344@drexel.edu, Phone: (215)-895-2268, Website: http://www.schweitzer-stenner.com

B. M. W.: E-mail: bryan.wong@ucr.edu, Phone: (951)-827-2163, Website: http://www.bmwong-group.com

\begin{abstract}
We investigate the UV absorption spectra of a series of cationic GxG peptides (where $\mathrm{x}$ denotes a guest residue) in aqueous solution and find that only a subset of these spectra show a strong dependence wtih temperature. To explore whether or not this observation reflects conformational dependencies, we carry out time-dependent density functional calculations for the polyproline II (pPII) and $\beta$-strand conformations in implicit and explicit water. We find that the calculated CD spectra for pPII can qualitatively account for the experimental spectra irrespective of the water model. The $\beta$-strand UV-CD spectra, however, requires the explicit consideration of water. Contrary to conventional wisdom, we find that both the $\mathrm{NV}_{1}$ and $\mathrm{NV}_{2}$ band are the envelopes of contributions from multiple
\end{abstract}


transitions that involve more than just the HOMOs and LUMOs of the peptide groups. A natural transition orbital analysis reveals that some of the transitions have a charge-transfer character. The overall manifold of transitions depends on the peptide's backbone conformation, peptide hydration, and side chain of the guest residue. Our results reveal that peptide groups, side chains, and hydration shells must be considered as an entity for a physically valid characterization of UV absorbance and circular dichroism. 


\section{Introduction}

Short peptides that contain only a few amino acid residues are generally assumed to be in a so-called random coil state. For individual residues, the generic Ramachandran plot suggests that they sample the entire sterically accessible space, which is considered to be nearly isoenergetic. With the exception of proline and glycine, this space and, thus, the conformational ensemble would be very similar for all amino acid residues. ${ }^{1-8}$ In such a case, it makes sense to assume that the random coil conformations can be represented by a single absorption spectrum. However, over the last 15 years, experimental and computational studies have revealed that individual residues differ with regard to the conformational sampling, with the conformational entropy of short peptides and disordered and unfolded proteins being significantly less than assumed. ${ }^{9-17}$ Recent results from MD and DFT computations have pointed to the same direction. ${ }^{18-24}$ All residues predominantly sample the upper left quadrant of the Ramachandran plot, and they differ mostly in terms of their population of the polyproline II (pPII) $\left(\varphi>-100^{0}, \psi>100^{\circ}\right)$ and $\beta$-strand region $\left(\varphi \leq-100^{0}, \psi>\right.$ $\left.100^{0}\right)$. The former is stabilized enthalpically while the latter is favored entropically. ${ }^{7}$

Besides NMR and vibrational spectroscopies, UV circular dichroism (UV-CD) has emerged as a suitable tool to distinguish between different conformational ensembles of short peptides and unfolded proteins. ${ }^{25-30}$ Owing to the preponderance of the sampling of pPII and $\beta$-strand-like conformations, ${ }^{10,31}$ all spectra exhibit a negative maximum around $190 \mathrm{~nm}$, the intensity of which reflects the pPII content of the distribution. If the pPII content is high, a weak positive maximum also appears in the region at $215 \mathrm{~nm}$. Generally, the intensities of both maxima decrease with increasing temperature owing to the increasing population of $\beta$-strand-like conformations. ${ }^{29,30,32} \mathrm{It}$ is widely assumed that the conformational changes involving a pPII $\leftrightarrow \beta$-strand transition lead exclusively to changes in the UV-CD signal due to an excitonic coupling mechanism. However, a recent computational analysis of the electronic transitions underlying the UV-CD spectra of the

cationic GAG in explicit water suggests that the electronic structure of pPII and $\beta$-strand are different in both the ground and the excited states of the peptide. ${ }^{33} \mathrm{We}$, therefore, wondered whether these differences are also reflected by the corresponding far UV absorption (UV-A) 
spectra.

While far UV-CD spectroscopy has been the spectroscopic technique of choice for the secondary structure analysis of proteins for over a period of 60 years (if we include its predecessor, optical rotary dispersion spectroscopy), ${ }^{34-36}$ the corresponding UV-A spectra have been mostly neglected after a period of exploration in the fifties and sixties of the last century. ${ }^{37-39}$ The neglect of this topic has several reasons. The UV-A spectrum of peptide/protein backbones generally displays one broad band with a peak in the region between 190 and $200 \mathrm{~nm}^{38}$ that overlaps with a broad absorption band of water. Compared with the respective UV-CD spectrum, its structure sensitivity is generally considered as somewhat limited. While UV-A spectra of $\beta$-sheets and $\alpha$ helices differ with regard to their respective molar absorptivities due to the hypochronism of the latter, the spectra of $\beta$-sheets and so-called random coil conformations were reported to be similar to each other. ${ }^{40}$ As a consequence, they do not appear as suitable tools for the analysis of unfolded peptides and proteins. Amino acid residues with sulfhydryl groups or unsubstituted amide bonds can contribute to the far UV-A spectra, whereas their contribution to the corresponding UV-CD spectrum does not seem to be significant. ${ }^{39}$ Overall, the lack of major fingerprints in the UV-A spectra of peptides makes it a less widely studied tool compared to UV-CD for structure analysis.

To check whether the UV-A spectra of statistical coil type conformations can still be distinguished, this study compares the temperature dependence of the UV absorption of a series of Gly-x-Gly tripeptides $(\mathrm{GxG})$, where the middle -x- residue is a non-aromatic residue guest in the minimal neighbor environment of glycine residues. To shed some light on the origin of the observed changes, we performed time-dependent density functional theory (TDDFT) calculations of UV-CD and UV-A spectra for a selected number of GxG peptides in explicit and implicit water. Recently, we showed that the explicit consideration of water is necessary to calculate the UV-CD spectrum of GAG in $\beta$-strand conformations. Our results revealed rather complicated electronic structures of the investigated peptides, which are inconsistent with the traditional excitonic coupling approach. ${ }^{33}$ Herein, we further show that (1) the background (water) corrected UV-A absorption spectra of most of the investigated GxG peptides depend on the temperature below 200 $\mathrm{nm}$, and (2) this temperature dependence depends on the choice of the host residue. Generally, our 
results shed new light on the charge-transfer character of electronic transitions that give rise to the well-studied UV-CD and UV-A spectra of peptides and proteins. Our results call for a critical assessment of theoretical models that have been used to model respective polypeptide and protein spectra over the last 70 years.

\section{Methods}

\section{Material}

All L-glycyl-x-glycine $(\mathrm{GxG}, \mathrm{x}=$ alanine, lysine, leucine, isoleucine, arginine, methionine, cystine, valine, aspartic acid, asparagine, and threonine) peptides were purchased from Genscript Corp. with $>98 \%$ purity and purified via dialysis in an aqueous $\mathrm{HCl}$ medium and subsequent lyophilization (Figure S1). For most of the $\mathrm{CD}$ and absorption measurements, the peptide was dissolved in a solution of $\mathrm{H}_{2} \mathrm{O}$ at a concentration of $0.01 \mathrm{M}$. The $\mathrm{pH}$ was adjusted to 2 with $\mathrm{HCl}$ for all experiments to allow a comparison with earlier spectroscopic work on the investigated tripeptides and to avoid interferences with $n \rightarrow \pi$ charge transfer transitions from the carboxylate end group to the neighboring peptide unit. ${ }^{41,42}$

\section{Ultraviolet Circular Dichroism and Absorption Spectroscopy}

UV-CD and absorption spectra were measured on a Jasco J-810 spectropolarimeter (Jasco, Inc.) purged with $\mathrm{N}_{2}$. The $0.01 \mathrm{M}$ sample was loaded into a $50 \mu \mathrm{m}$ International Crystals Laboratories (ICL) cell. The UV-CD spectra reported in this study have been measured earlier; however, the specific room temperature spectra shown in this study were not reported in our earlier publication. ${ }^{30}$ To correct for the background (water and instrument), we measured the spectra of water with the same setup as a function of temperature. In addition, we repeated the measurement 
of the absorption spectra of cationic GAG, GVG, and double protonated GDG to improve the signal to noise of these particular spectra and to check for the reproducibility of the earlier recorded spectra. All spectra were measured between 180 and $300 \mathrm{~nm}$ with a $500 \mathrm{~nm} / \mathrm{min}$ scan speed, a $1 \mathrm{~s}$ response time, a 0.05 data pitch, and a $5 \mathrm{~nm}$ bandwidth. Earlier measured spectra were taken from 10 to $85{ }^{\circ} \mathrm{C}$ with $5{ }^{\circ} \mathrm{C}$ increments using a Peltier controller (model PTC-423S). All spectra were corrected using appropriate background subtraction. More recent recordings of the absorption spectra of GAG, GVG, and GDG were carried out between 10 and $80{ }^{\circ} \mathrm{C}$ in increments of $10{ }^{\circ} \mathrm{C}$.

\section{TDDFT Calculations}

Quantum mechanical calculations were carried out using the Gaussian 09 program package. ${ }^{43}$ We optimized the geometry of the peptides after obtaining the coordinates from the Supplementary Information reported by Ilawe et al. ${ }^{44}$ These calculations were followed by a harmonic frequency analysis to assess whether the stationary points obtained were local minima. After this, we performed excited-state TDDFT calculations to obtain the UV absorption and electronic circular dichroism spectra of the peptides. Previous studies ${ }^{45-47}$ have utilized TDDFT calculations to examine the underlying physics of the CD spectra of model peptides and proteins in their folded state. A similar investigation of unfolded peptides was performed in our previous work. ${ }^{33}$ This work expands the previous study to include other variants of short peptides. As discussed before, we recently performed DFT calculations on cationic GAG peptide in implicit and explicit water to obtain the energetics of their pPII and $\beta$-strand conformations. The present study considers the optimized geometry of these two conformations of $\mathrm{GxG}(\mathrm{x}=\mathrm{A}, \mathrm{I}, \mathrm{L}$, and V) peptides for subsequent TDDFT calculations at the $\omega$ B97X-D/cc-pVTZ level of theory to calculate the UV-CD and corresponding UV-A spectra of GxG in implicit and explicit water. To add a GxG peptide with a charged side chain to this list, we first optimized the geometry of pPII and $\beta$ strand conformation of GRG in implicit and explicit water $\left(10 \mathrm{H}_{2} \mathrm{O}\right)$ and subsequently calculated absorption and UV-CD spectra with TDDFT. The water positions in explicit water models were chosen based on a previous study of ours, ${ }^{44}$ which was based on MD/DFT studies of hydrogen- 
bonded water molecules. ${ }^{48}$ We specifically chose the $\omega$ B97X-D/cc-pVTZ functional for our studies since it contains an asymptotically-correct (range-separated) portion of nonlocal exchange in conjunction with dispersion corrections, which are essential for accurately predicting chargetransfer excitations and hydrogen-bonding interactions, respectively. It is also important to note that prior work by Neto et al. ${ }^{49}$ has shown that the optimal range-separated parameter, $\omega$, required to accurately predict excited-states in the explicit solvent is 0.25 , which is already close to the default value of $\omega=0.2$ used in the $\omega \mathrm{B} 97 \mathrm{X}-\mathrm{D} / \mathrm{cc}-\mathrm{pVTZ}$ functional. We showed in our previous work that (1) changing the range-separation value, (2) using different exchange-correlation functionals, (3) utilizing a larger basis set (such as aug-cc-pVTZ), and (4) altering the positions of explicit water molecules, did not change the spectra significantly within the relevant wavelength range (Fig. S8).

To explore how the UV-CD spectra change when more excited-state electronic transitions are included, we also performed additional TDDFT calculations with 50 excited-state transitions. We did not observe any difference between the UV-CD spectra computed with 30 and 50 excitedstate transitions in the relevant wavelength range (Fig. S9). Therefore, we calculated the absorption and the UV-CD spectra within the relevant wavelength range at the $\omega \mathrm{B} 97 \mathrm{X}-\mathrm{D} / \mathrm{cc}-\mathrm{pVTZ}$ level of theory and 30 excited state transitions for this work. We followed the same method reported in our previous study ${ }^{33}$ to obtain UV-CD and absorption spectra. The spectral region covered by our calculation comprises two bands that are generally termed $\mathrm{NV}_{1}$ and $\mathrm{NV}_{2}$. Experimentally, $\mathrm{NV}_{1}$ appears at around $190 \mathrm{~nm}$ in peptide and protein spectra and is generally assigned to a $\mathrm{HOMO} \rightarrow \mathrm{LUMO}$ transition of the peptide $\pi$-electron system. $\mathrm{NV}_{2}$ generally appears at much lower wavelengths and is thought to be mostly assignable to the $\mathrm{C}=\mathrm{O}$ bond. As we will show in this paper, the electronic underpinnings of the two bands are more complex. The Supporting Information contains optimized geometries of all GxG peptides, detailed absorption and UV-CD spectra with the underlying transitions, comparisons between spectra of different conformations, lists of $\mathrm{NV}_{1}$ and $\mathrm{NV}_{2}$ transition peaks, and tables with a detailed list of the first five excited states.

\section{Results and Discussion}


This section of the paper is organized as follows. First, we show a selected number of GxG UV-A absorption spectra (c.f. Figure S1) taken at different temperatures between 10 and $70{ }^{\circ} \mathrm{C}$. We augment these experiments with a short description of the room temperature UV-CD of the GxG peptides for which we performed the TDDFT calculations in implicit and explicit water $(\mathrm{x}=$ A, V, I, L, and R). Next, we describe the calculated UV-CD spectra and the underlying electronic structure followed by a description of the corresponding absorption spectra. Third, we compare the calculated and experimentally observed spectra. The final paragraph uses NTO figures to explore the character of the main transitions that underlie the calculated UV-CD and UV-A spectra. We conclude by comparing our results with earlier analyses of peptide/protein UV-A spectra that highlights the implications of our results.

\section{Experimental UV absorption and circular dichroism spectra.}

Figure 1 shows the UV-A spectrum of cationic GAG in water at a $\mathrm{pH}$ level of 2 without (left) and with (right) background subtraction taken at four different temperatures between 10 and $70{ }^{\circ} \mathrm{C}$. We display only some of the recorded spectra to facilitate the reading of these and subsequent figures. Figure 2 shows the corresponding UV-A spectra of GVG, GIG, GLG, and GRG after water background subtraction. The spectra prior to the background subtraction should be considered as a superposition of both the water absorption spectrum and the instrument's transfer function. 


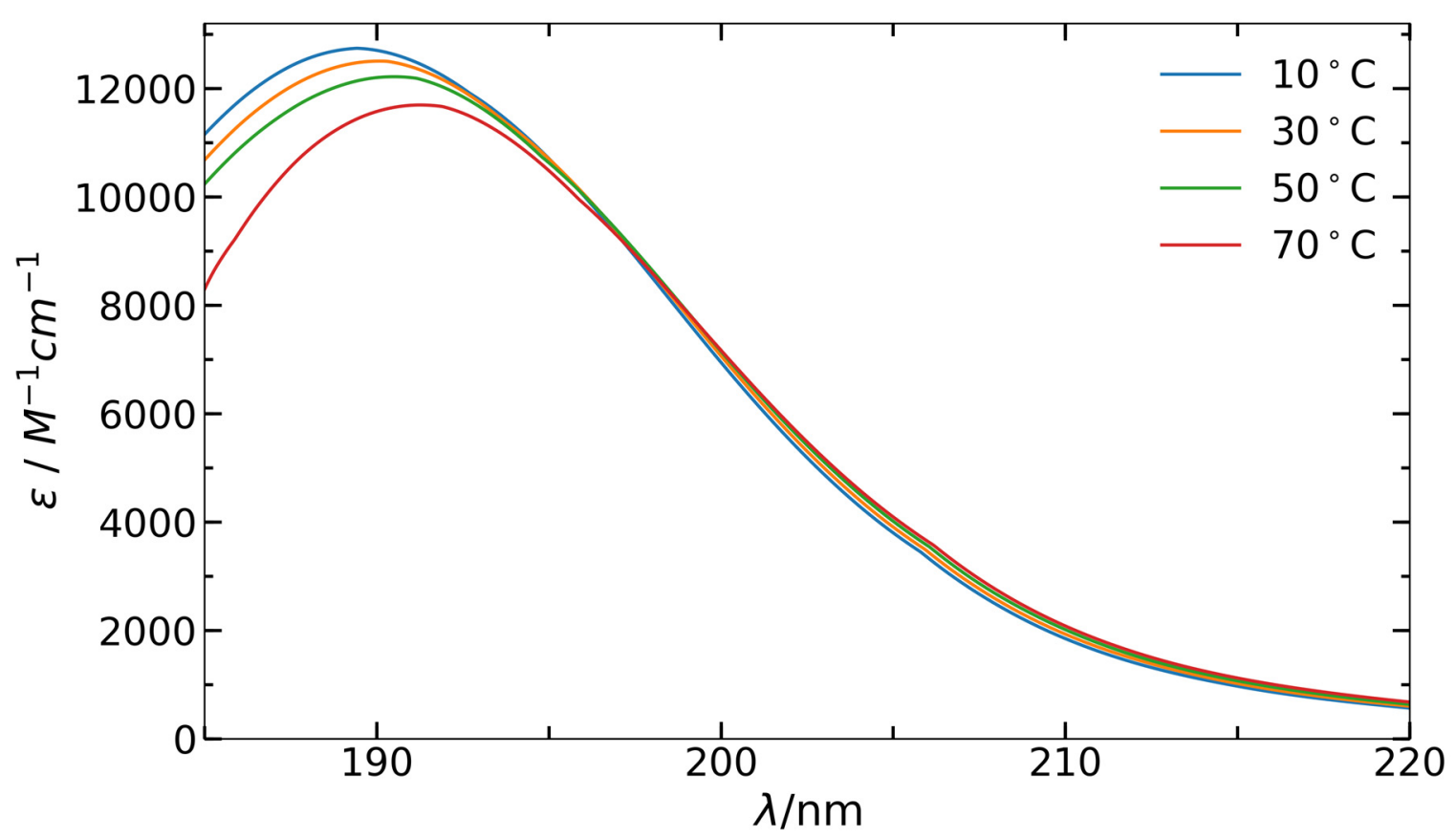

Figure 1: UV-absorption spectra of cationic $\mathrm{GAG}$ in water at $\mathrm{pH}=2$ after background subtraction taken at $10,30,50$, and $70^{\circ} \mathrm{C}$.

As shown in Figure 1, the uncorrected GAG UV-A spectrum has a shoulder at $195 \mathrm{~nm}$ that corresponds to the maximum of the absorption band at $190 \mathrm{~nm}$ in the background-corrected spectrum. The uncorrected spectra seem to suggest that the absorptivity below $190 \mathrm{~nm}$ increases with temperature. However, the spectra obtained after an appropriate background subtraction show the opposite effect, with clearly decreasing maxima as a function of increasing temperature. The more aliphatic GIG and GLG peptides show a similar behavior (Figure 2, lower panels). The temperature dependence of the GLG (Figure 2, lower right panel) is slightly more pronounced than that of GAG and GIG. Generally, for all aliphatic residues, the band maximum shifts to the red, and the absorptivity decreases on the high energy side. GVG was found to behave differently in that the spectra solely indicate a redshift without much loss in oscillator strength. The absorption band of GRG depicts a much larger intensity than the corresponding bands of any of the yet discussed peptides, which is indicative of side-chain contributions. ${ }^{31}$ The temperature dependence of the band is weak, with an overall redshift of the band, similar to what we observed for GVG. 

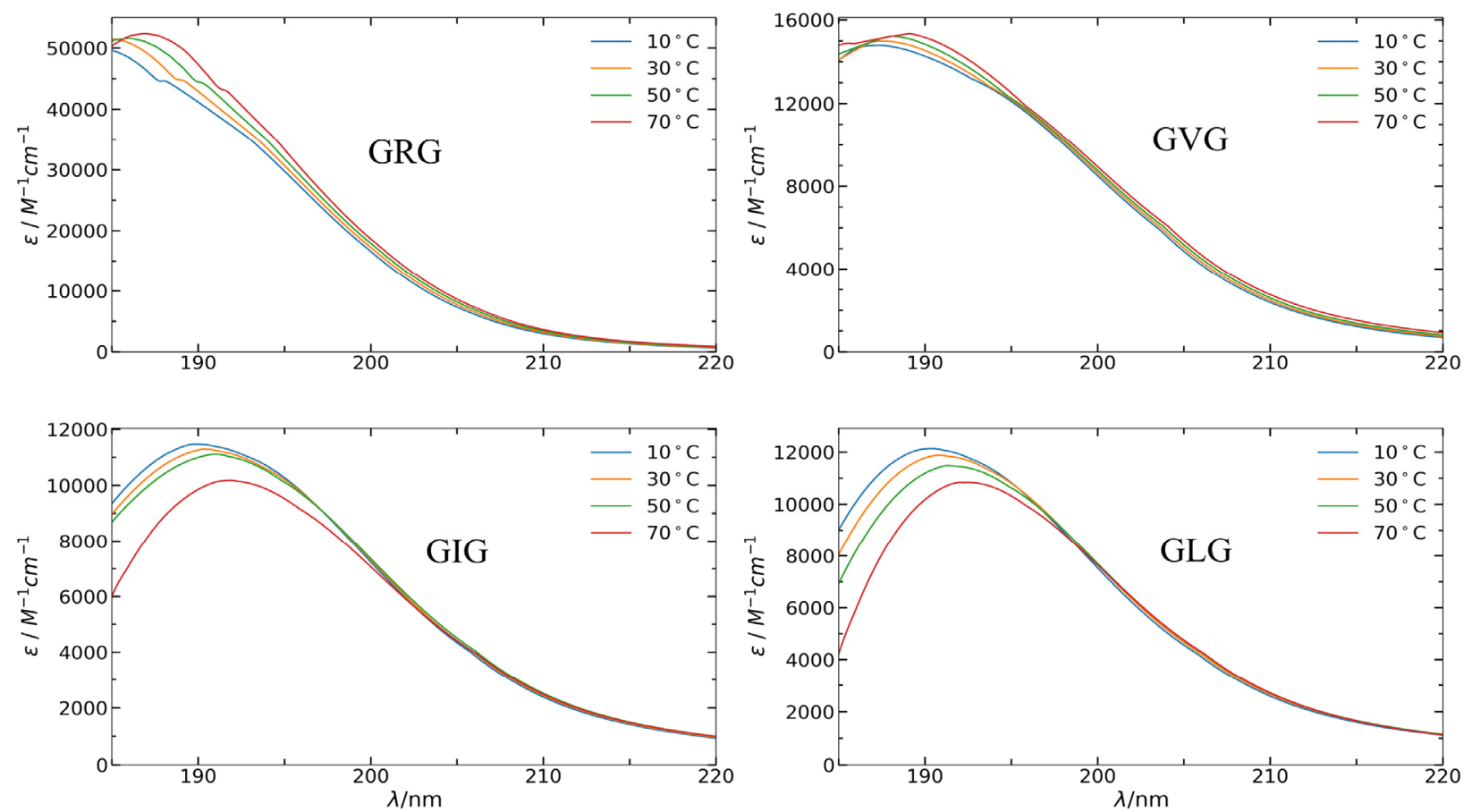

Figure 2: UV absorption spectra of GRG, GVG, GIG, and GLG after water/background subtraction measured at the indicated temperatures.

To examine the effect of side-chain interactions, we additionally measured the absorption spectra of selected GxG peptides with non-aliphatic side chains. The background-corrected spectra of GKG, GNG, GDG, GMG, GTG, and GCG are all shown in Figure S2. GNG and GKG are similar to GLG and exhibit very similar temperature dependencies. GDG shows a normal absorptivity, but a very weak and barely detectable temperature dependence. The temperature dependences of the GTG, GMG, and GCG absorbance shown in Figure S2 are all weak. For GMG and GCG, this is most likely due to large side chain's contributions assignable to the SH and SC bonds of their respective side chains. The absorption of GTG results from backbone transitions. Therefore, the corresponding oscillator strength is comparable with those of GKG and GLG.

It is evident from our experimental data that some of the absorption spectra of the investigated $\mathrm{GxG}$ peptides depend on temperature, while others are nearly temperature independent. The absence of a pronounced temperature dependence for GTG, and to a lesser extent GDG and GVG, rules out the possibility that the observed changes reflect intrinsic properties of 
individual peptides and their interactions with the solvent, as observed for chromophores in heme proteins and solvents. ${ }^{50,51}$ Therefore, to gain more insight into the amino acid-specific temperature dependence of the UV-A spectra, we turn to temperature-dependent UV-CD analysis.

UV-CD spectra of the investigated peptides have been reported for selected temperatures in an earlier paper. ${ }^{30}$ Here, we show the yet unpublished room temperature spectra $\left(20^{\circ} \mathrm{C}\right)$ of GAG, GVG, GIG, GLG, and GRG (Figure 3) for a comprehensive comparison with TD-DFT calculations described below. The spectra of GAG, GLG, and GRG depict a very negatively biased positive couplet, which is indicative of a substantial sampling of pPII conformations,${ }^{26}$ while the spectra of GVG and GIG show a less intense negative maximum and no clearly discernable positive maximum. In agreement with other experimental data, the latter suggests substantial sampling of $\beta$-strand-like conformations. ${ }^{52,53}$

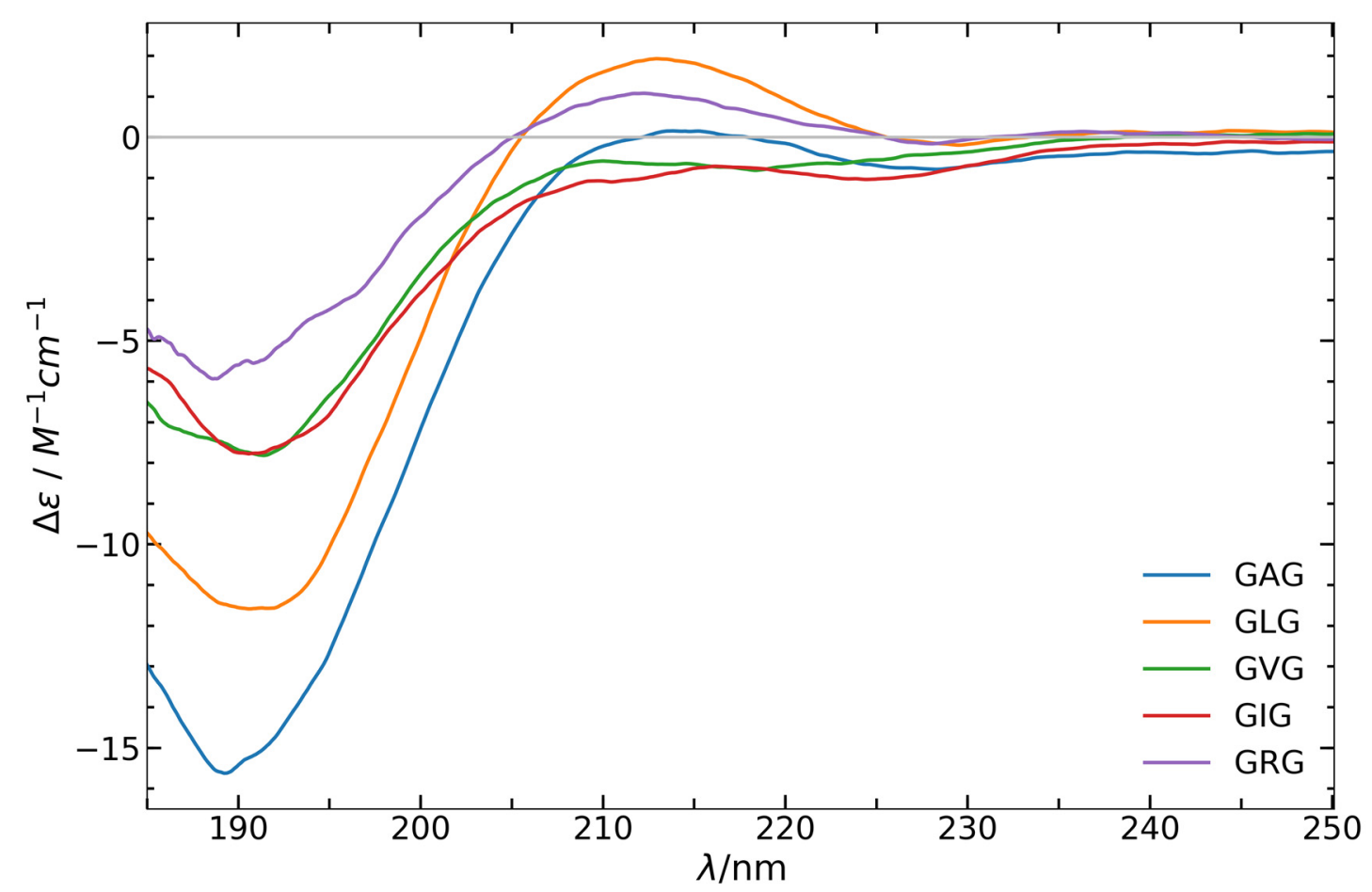

Figure 3: UV-CD spectra of cationic $\mathrm{GxG}(\mathrm{x}=\mathrm{A}, \mathrm{L}, \mathrm{V}, \mathrm{I}$, and $\mathrm{R})$ in water measured at room temperature $\left(20^{\circ} \mathrm{C}\right)$. Individual dichroism values of these spectra were reported by Toal et al. (cf. Ref. 16) 


\section{TDDFT calculations of CD and absorption spectra.}

TDDFT calculations were carried out for five peptides with side chains, i.e., GAG, GVG, GIG, GLG, and GRG in explicit and implicit water for the respective pPII and $\beta$-strand conformations. The explicit water calculation considered 10 water molecules that are either hydrogen-bonded to the peptide and are inserted in a hydrogen-bonding network of water molecules. The structures for the four aliphatic peptides were recently obtained by geometry optimization. ${ }^{44}$ Additionally, we optimized the geometries of cationic GRG for pPII and $\beta$-strand conformations in explicit $\left(10 \mathrm{H}_{2} \mathrm{O}\right)$ and implicit water to explore a situation in which the side chain transitions can be expected to contribute to the measured absorptivity below $180 \mathrm{~nm}$ to a significant extent. We calculated the CD as well as the absorption spectra for these peptides and both water models. Figures 4 and 5 depict the respective spectra obtained with the explicit water model. Figure S3 and S4 compare the spectra calculated with explicit and implicit water as well as the underlying electronic transitions. As usual, the energies of electronic transitions are overestimated by our TDDFT calculations. Hence the wavelength range in Figures 4, 5, S3, and S4 are all blueshifted relative to that of the experimental spectra in Figure 3. Experimental limitations rule out measuring any absorption and CD spectra below $185 \mathrm{~nm}$.

The following observations are noteworthy. First, all explicit water calculations yielded the classical feature of pPII, namely negatively biased couplets with a negative maximum at 175 and a positive maximum at $210 \mathrm{~nm}$. For $\beta$-strand conformations in explicit water, we observed nearly equally intense positive and negative maxima at ca. 185 and $205 \mathrm{~nm}$, respectively. For GLG, the two extrema are very weak. None of the calculations with implicit water reproduce this couplet signal. This underscores the earlier reported notion that explicit water must be considered to account at least quantitatively for the spectra of $\beta$-strand conformations. ${ }^{33}$

The influence of explicit water and geometric conformation on the electronic structure can 
be inferred from dissimilar rotational strengths of the $\mathrm{NV}_{1}$ transition peaks obtained for the $\beta$ conformations (Figure S3). The implicit water calculation yielded a series of transitions with mostly weak negative rotational strengths. In explicit water, one of these transitions gains a positive rotational strength, giving rise to the positive maximum that is characteristic of the experimental $\beta$-strand CD spectra. Differences between the results of the two water models are less pronounced for $\mathrm{pPII}$, where the negative maximum associated with the $\mathrm{NV}_{1}$ band comprises several transitions with negative rotational strengths (Figure S4).

The calculated absorption spectra (see Figure 5 for explicit water and Figures S6 and S7 for implicit water) display two major absorption bands, one around $140 \mathrm{~nm}$ and another between 170 and $180 \mathrm{~nm}$. Conventionally, they would be interpreted as indicating the peptide groups' $\mathrm{NV}_{2}$ and $\mathrm{NV}_{1}$ transitions from the $\pi$-orbital HOMO to the two lowest $\pi^{*}$-orbital LUMOs, ${ }^{38,39}$ but as we show below, this assignment would be way too simplistic. However, for the sake of conciseness, we term the corresponding bands $\mathrm{NV}_{1}$ and $\mathrm{NV}_{2}$ in the following. 

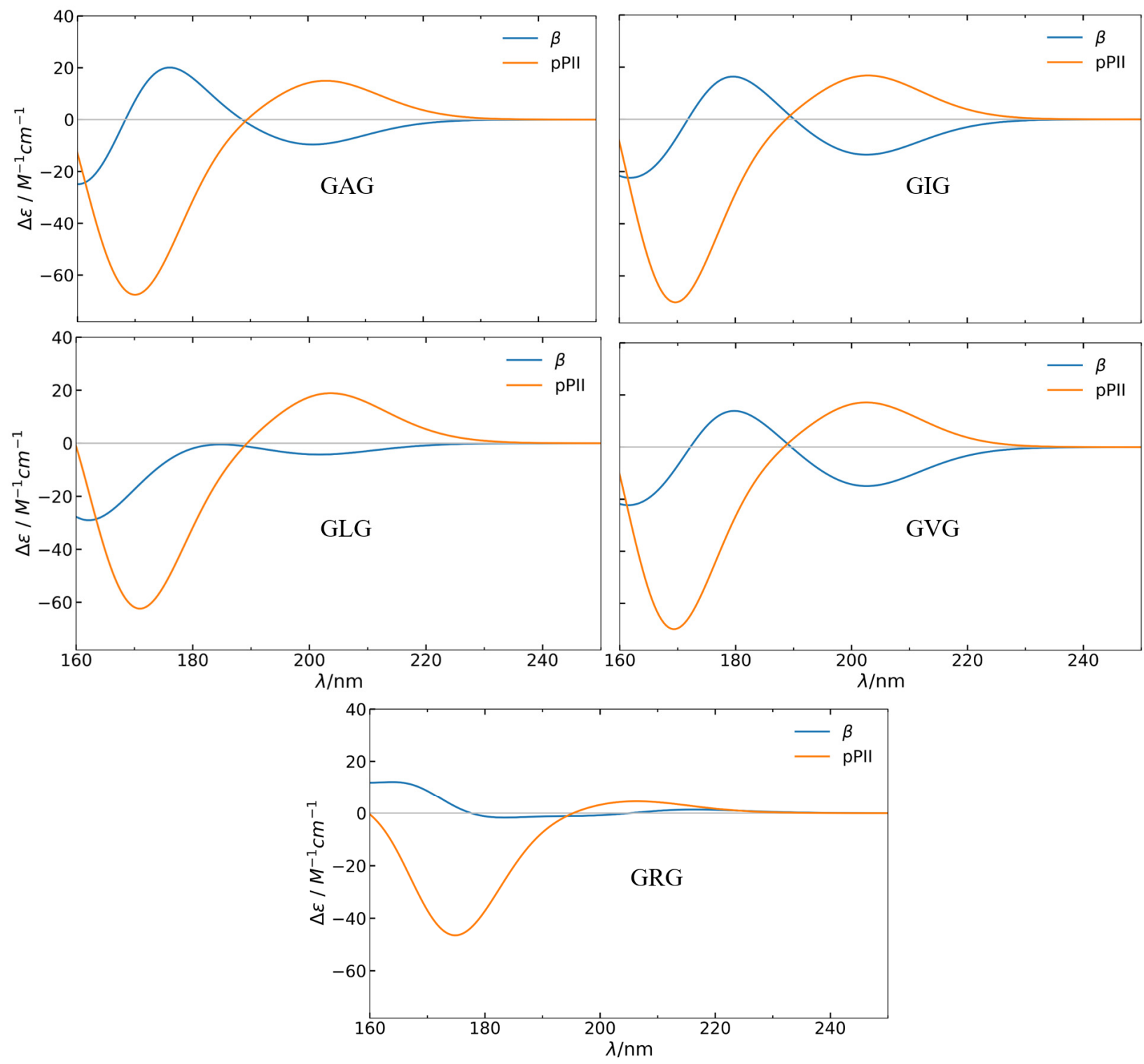

Figure 4: Calculated UV-CD spectra of indicated GxG peptides in their $\beta$ and pPII conformation with explicit water.

As can be inferred from Figures 5, the $\mathrm{NV}_{1}$ bands of $\beta$ strands exhibit higher oscillator strengths than their respective pPII conformations for the aliphatic peptides GAG, GIG, GLG, and GVG in explicit water. Only for GRG does the pPII intensity of $\mathrm{NV}_{1}$ exceed that of the corresponding $\beta$-conformation. It is also noteworthy that the $\beta$ conformations of GVG, GIG, and GLG exhibit lower transition energies for their $\mathrm{NV}_{1}$ transition peaks than the corresponding pPII, with the average difference of $1.2 \mathrm{~nm}$ (Table S2-S4). 
Figure 5 shows the calculated distributions of oscillator strengths associated with bands $\mathrm{NV}_{1}$ and $\mathrm{NV}_{2}$ in the $\mathrm{pPII}$ and $\beta$-strand conformations of the investigated $\mathrm{GxG}$ peptides $(\mathrm{x}=\mathrm{A}, \mathrm{I}$, L, V, and R) obtained with the explicit water model. The corresponding distributions obtained with the implicit water model are depicted in Figure S7. In the absorption spectra of the $\beta$-strand conformations of the aliphatic peptides, the respective transitions into the fourth excited state dominate the $\mathrm{NV}_{1}$ peak region. For the corresponding pPII conformations, transitions into the fourth and the fifth excited states account for most of the $\mathrm{NV}_{1}$ absorption peak (cf. Table S1-S5 in the SI). The $\mathrm{NV}_{2}$ band of the aliphatic peptides is just the envelope of a rather large number of electronic transitions (Figures 5 and Figure S7). The influence of the water model on the calculation of the $\mathrm{NV}_{2}$ band is significant. First, the explicit consideration of water molecules leads to an increase of transitions and thus an increase of the overall oscillator strengths. Second, the dispersion of $\mathrm{NV}_{2}$ transitions is significantly more pronounced in the pPII conformations. As a consequence, the corresponding $\mathrm{NV}_{2}$ bands appear sharper with higher peak intensities in the $\beta$ strand conformations compared with respective bands of the pPII conformations. 


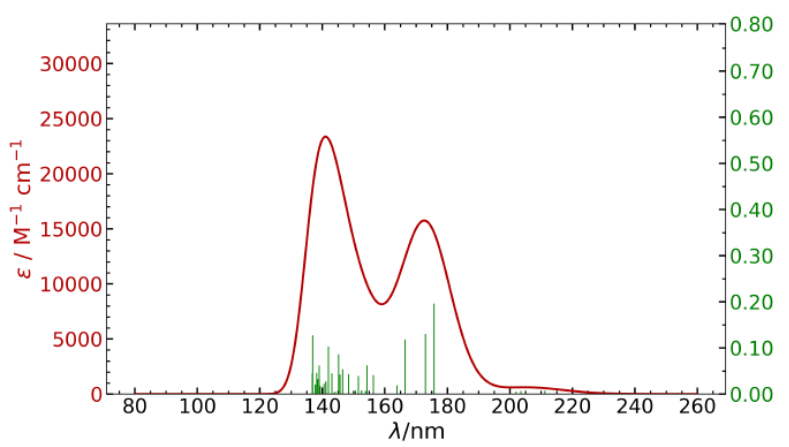

GAG pPII

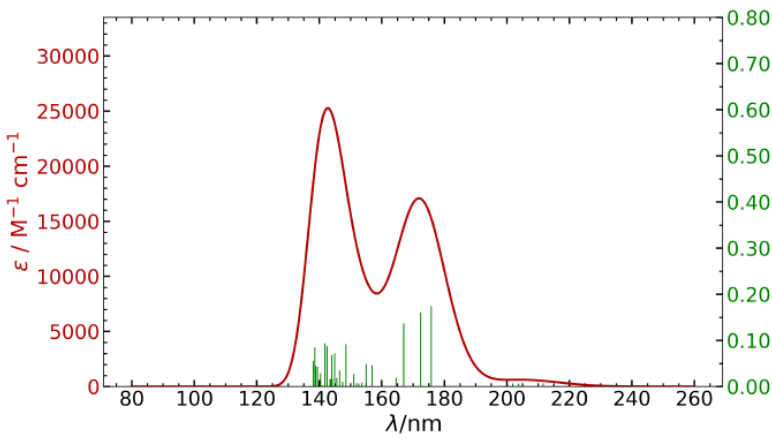

GIG pPII

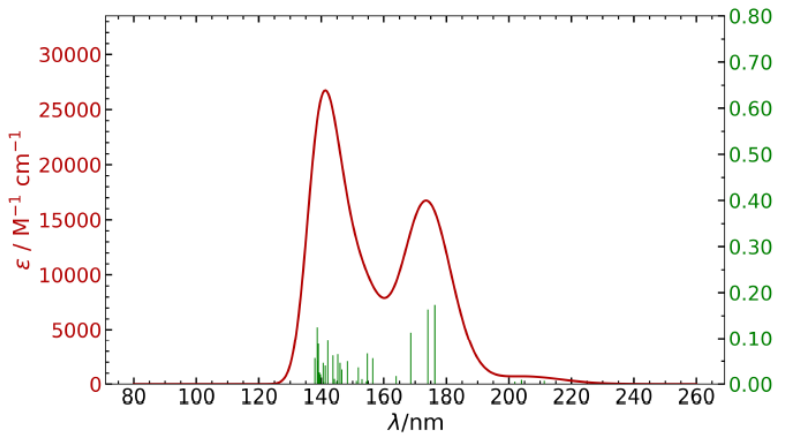

GLG pPII

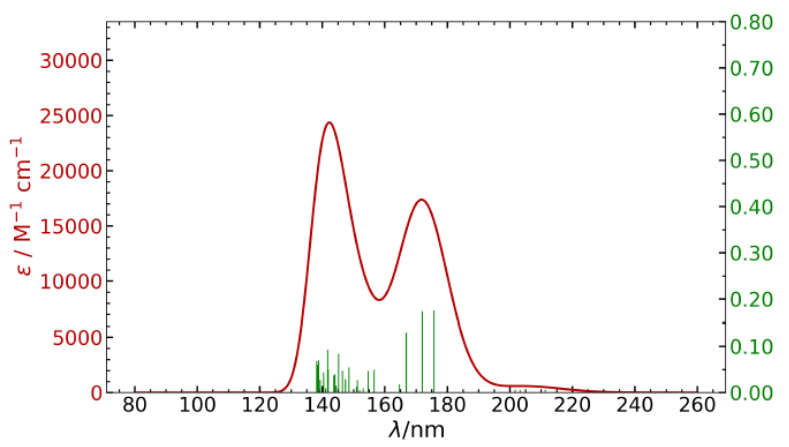

GVG pPII

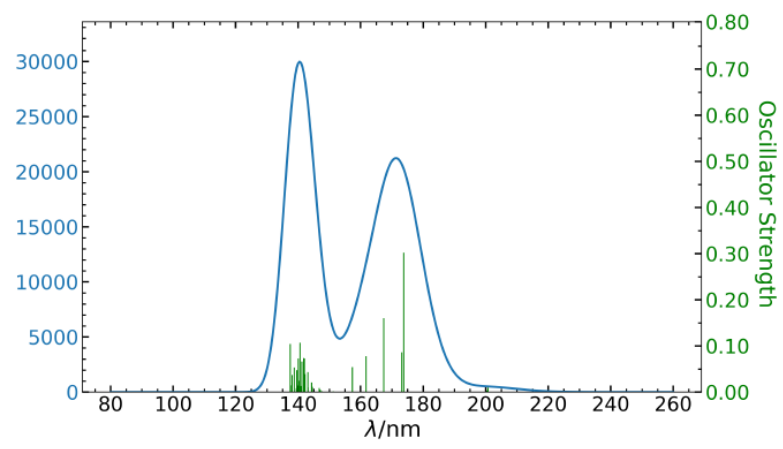

GAG $\beta$

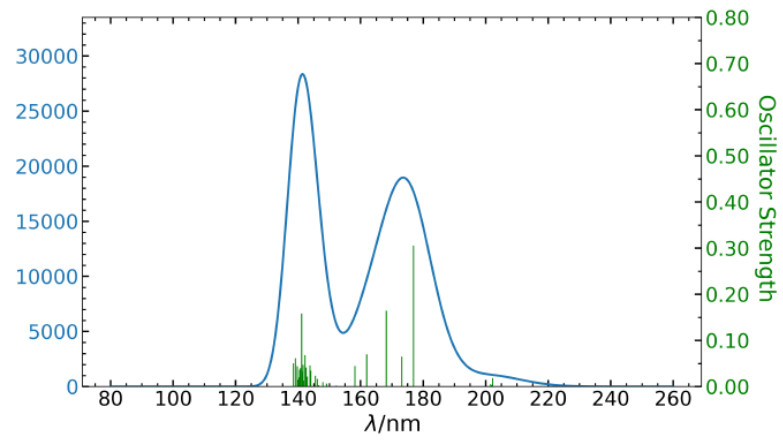

GIG $\beta$

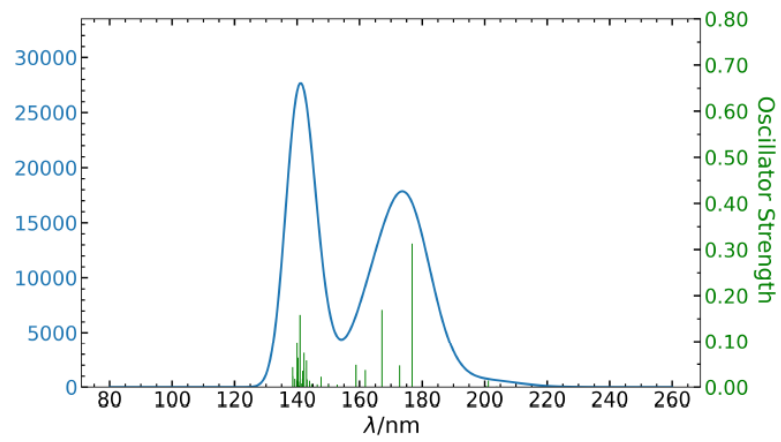

GLG $\beta$

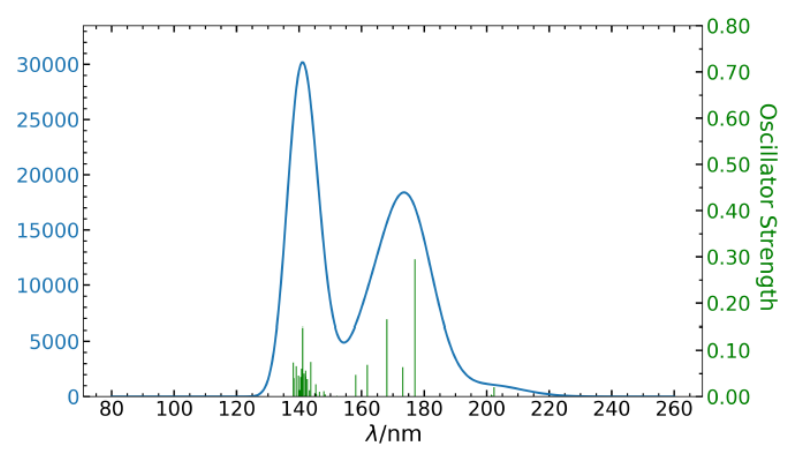

GVG $\beta$

Figure 5. The calculated distributions of oscillator strengths associated with bands $\mathrm{NV}_{1}$ and $\mathrm{NV}_{2}$ in the pPII and $\beta$-strand conformations of the indicated GxG peptides ( $x=A, I, L, V$, and $R$ ) obtained with the explicit water model. The sticks represent the underlying electronic transitions. 
The calculated absorption spectra of both the $\beta$ and pPII conformation of GRG in implicit water show a peak at around $160 \mathrm{~nm}$ and a shoulder at around $142 \mathrm{~nm}$ (Figure S7). The shoulder disappears when the absorption spectra are calculated with the explicit water model, and the absorption spectra show distinct peaks at around $168 \mathrm{~nm}(162 \mathrm{~nm})$ and $148 \mathrm{~nm}(144 \mathrm{~nm})$ for the $\beta$ (pPII) conformation (Figure 5). The $\mathrm{NV}_{1}$ peaks for both the $\beta$ and pPII conformation have excitedstate transitions with a large oscillator strength (0.50). None of the calculated absorption spectra of the other GxG peptides showed such a large oscillator strength for a transition into any excited states.

Comparison of Experimental and Computational Spectra. In view of the apparent superiority of the explicit water model regarding the calculation of the CD spectra of $\beta$-strand conformations, we solely compare experimental and explicit water spectra. The pPII spectra of all aliphatic peptides are very similar, which suggests that the rotational strength of this conformation does not depend very much on the nature of the side chain. This is an important finding for any quantitative analysis of the UV-CD spectra of unfolded peptides. The situation is somewhat different for the $\beta$-strand conformation. The corresponding spectra are similar for GAG, GIG, and GVG, while the rotational strength of GLG is much less. These results suggest that the different intensities of the negative maxima in Figure 3 are a good indicator of pPII propensities. Our computational results also explain why the experimental spectra of GAG and GLG are very similar even though the pPII propensity of the latter $(0.56)$ is significantly lower than that of the former $(0.79),{ }^{14}$ since a weaker positive maximum of the $\beta$-strand leads to a more intense negative maximum of $\mathrm{pPII} / \beta$ mixtures. It should be noted that the absolute dichroism displayed by the calculated spectra indicate a much larger rotational strength than the experimental ones. However, it has to be noted as well that the respective rotational strengths that emerged from the calculations with the implicit water model are even larger to a significant extent (Tables S6-S25). This observation suggests that the involvement of water molecules decreases the rotational strength, and one could, therefore, expect that calculations with an even larger number of water molecules 
would tune the CD signals into the correct order of magnitude.

At first glance, the calculated UV-A spectra do not seem to account for the observed temperature dependence of the $\mathrm{NV}_{1}$ band. For all four peptides, they indicate a more intense band in the $\beta$-strand conformation. The observed temperature dependence suggests the very opposite behavior. However, a closer analysis of the data suggests a different picture. First, for reasons described above, one has to take into account that the $\mathrm{NV}_{2}$ bands of the $\beta$-strand conformation are significantly sharper than those of the corresponding pPII state, which leads to a drop of intensity between the two bands. This is fully consistent with the observed intensity loss at the high energy site of the $\mathrm{NV}_{1}$ band. Second, a comparison of the $\mathrm{NV}_{1}$ oscillator strengths calculated with implicit and explicit water reveals important differences that reflect the pivotal role of hydration water. For GAG, GIG, and GVG, the combined oscillator strength of transitions 4 and 5 of the respective $\beta$ strand conformations were calculated to be a factor of 1.4-1.5 larger in the implicit than in the explicit water spectra. The difference between the corresponding pPII spectra is much less pronounced (ca. 1.1). GRG is a special case owing to side chain contributions that apparently make the contributions to the $\beta$-strand spectrum comparable for both models. Our results suggest that explicit water reduces the $\mathrm{NV}_{1}$ oscillator strengths mostly in the $\beta$-strand conformation. This effect is very likely to be more pronounced for a peptide with a complete hydration shell. As a consequence, the overall $\mathrm{NV}_{1}$ oscillator strength of the $\beta$-strand would become less than that of the respective pPII conformation, in agreement with our experimental results. Therefore, the temperature dependence of the UV absorption spectra of the investigated peptide reflects the degree by which water affects the electronic transitions in the $\beta$-strand. Furthermore, it is safe to infer a concomitant increase in the oscillator strength of $\mathrm{NV}_{2}$ from our results. At the current level of theory, it is not possible to quantitatively interpret the temperature dependence of the investigated GxG peptides. Only for protonated GDG, available experimental data provides us 
with an explanation of the very weak temperature dependence of the UV-A (Figure S2). As shown by Rybka et al., ${ }^{54}$ the conformational distribution of the central $\mathrm{D}$ residue of this peptide at room temperature is dominated by $\beta$-strand conformations and various turn-like conformations. Only $20 \%$ of the peptides sample pPII. As a consequence, a further increase of the $\beta$-strand population should not cause a significant temperature dependence of the UV-A, particularly because the turnlike content of the Ramachandran distribution is nearly temperature independent. ${ }^{21}$

\section{NTO analysis}

The contribution of peptide and terminal groups as well as side chains and hydration water of GxG peptides to NV1 transitions can be obtained by a natural transition orbital (NTO) analysis, which is based on the singular value decomposition of the 1-particle transition density matrix. The resulting frontier orbitals can represent any one-electron property associated with the electronic transition. As such, NTOs provide a compact representation of the orbitals and offer a convenient way to visualize excitations via occupied hole and virtual particles. The NTO analysis was also employed to determine the difference between the electronic structure of pPII and $\beta$ of investigated GxGs based on the nature of the excitations, which results in the observed absorption and UV-CD spectra. An NTO analysis imparts further impetus in characterizing the dichotomy in the spectra for $\beta$ and pPII conformations. The influence of water and the backbone conformation on the electronic structure of GxGs can be inferred from Figures 6-10 for explicit and Figures S10-S14 for implicit water. These figures depict the NTOs that can be attributed to the dominant transitions. The influence of explicit water and the backbone conformation can be illustrated by a comparison of corresponding NTOs. The transition into the fifth excited state gives rise to the $\mathrm{NV}_{1}$ band obtained for the pPII conformation of GAG (Figure 6). The particle is mostly delocalized across the two peptide bonds, while the hole is delocalized mostly on the N-terminal peptide group. For GAG in the $\beta$-strand conformation, the particle associated with the most intense 4 th transition is mostly delocalized across the two peptide groups. The corresponding transition of the hole to the particle involves the transfer of some electron density from the alanine residue to the peptide 
groups. For GIG, the visualized NTOs associated with the $\mathrm{NV}_{1}$ band of the pPII conformation suggest that particles are mostly localized at the carboxylate group while the hole is predominantly localized at the $\mathrm{C}$-terminal peptide group. In the corresponding $\beta$-strand conformation, the particle is delocalized over both peptide groups, whereas the hole is mostly localized at the N-terminal peptide bond (Figure 7). In the case of GLG, transitions into excited states 4 and 5 both contribute to the $\mathrm{NV}_{1}$ band of the pPII conformation. An analysis of the hole and particle for the 4 th excited state shows that the hole is localized at the carboxylate group, while the particle resides at the Cterminated peptide bond. The 5th excitation shows a hole slightly localized at the leucine residue and predominantly at the N-terminated peptide bond, whereas the particle is delocalized across both the peptide bonds. A 3rd transition (number 21) contributes significantly to the $\mathrm{NV}_{1}$ absorption. Both the hole and particle are delocalized over the peptide backbone. The hole exhibits some minor contributions due to the side chain. For the respective $\beta$-strand conformation, the NTO analysis yields a different picture. The particle is delocalized over the ammonium group and the adjacent peptide bond with some admixture from the $\mathrm{C}_{\alpha}-\mathrm{C}^{\prime}$ bond, whereas the hole is localized on the leucine residue. Hence, this transition again has a charge-transfer character. We wondered whether this was a very peculiar character of the electronic transitions that leads to the very weak CD signal of this conformation. For the pPII conformation of GVG (Figure 9), both the 4th and 5th excitations contribute to the $\mathrm{NV}_{1}$ band. The hole in the 4th excited state is localized at the carboxylate group, whereas the particle resides at the C-terminal peptide bond group. For the 5 th excitation, the hole is mostly localized on the valine residue, and the particle is delocalized across the peptide bonds. Some of the NTOs associated with the $\mathrm{NV}_{1}$ band of the $\beta$-strand conformation of GVG are delocalized over both the peptide group and the valine residue between them. The hole in the 4th excited state is delocalized over both the peptide groups while the particle is localized at the ammonium group. 
(a)

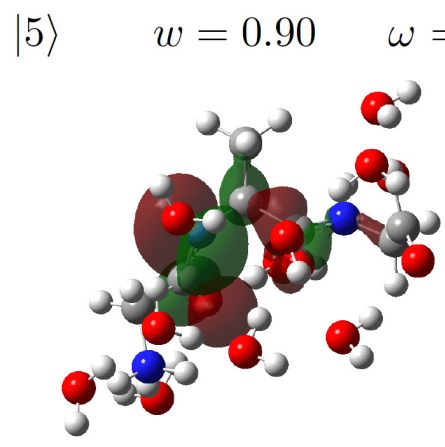

Hole $\lambda=172.98 \mathrm{~nm} \quad f=0.13$

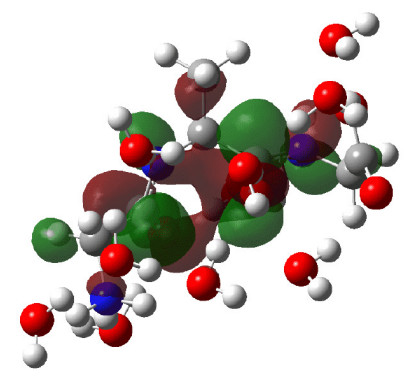

Particle

(b)

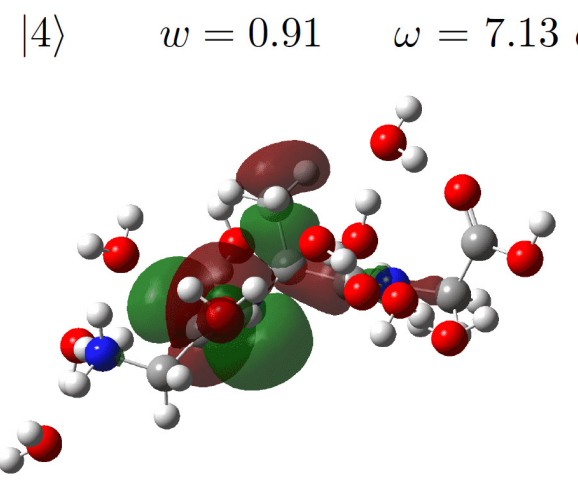

Hole

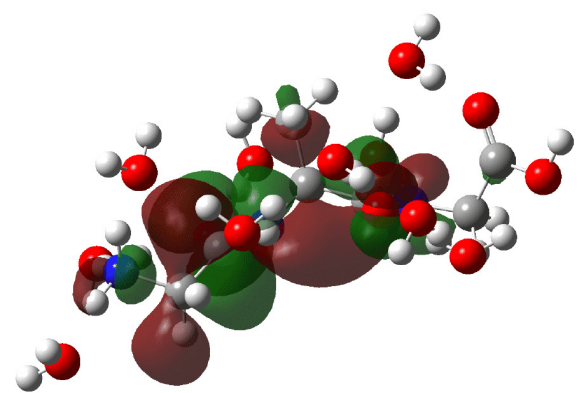

Particle

Figure 6. Natural Transition Orbitals (NTOs) of GAG in the (a) pPII and (b) $\beta$ conformations. The panels give the excited state number, associated eigenvalue $(w)$, transition energy $(\omega)$, wavelength $(\lambda)$, and oscillator strength $(f)$. 
(a)

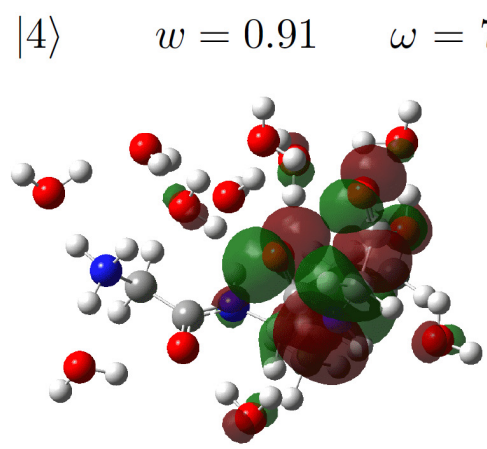

Hole $\lambda=175.80 \mathrm{~nm} \quad f=0.17$

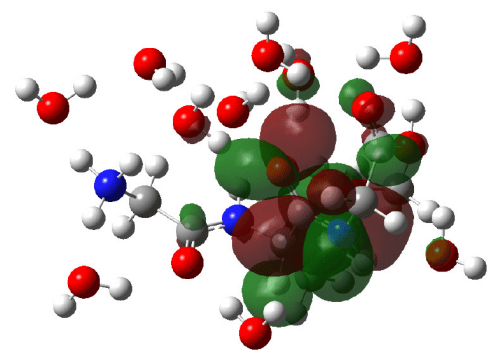

Particle

(b)

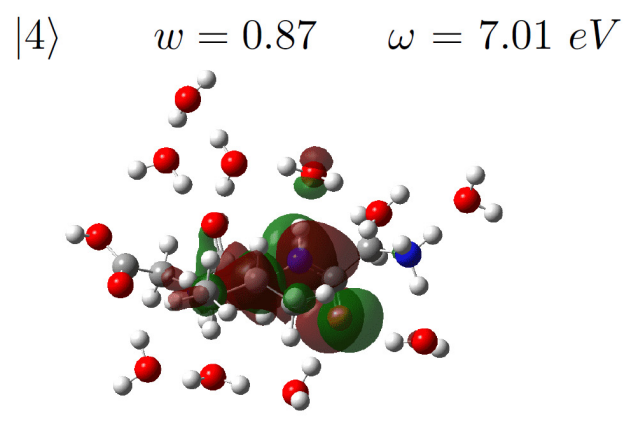

Hole

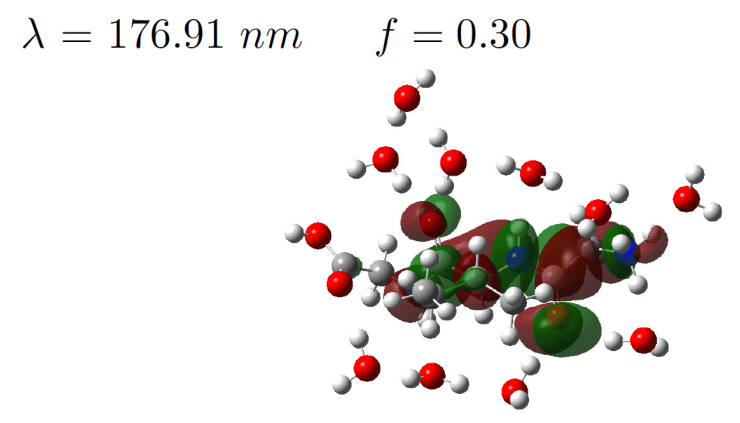

Particle

Figure 7. Natural Transition Orbitals (NTOs) of GIG in the (a) pPII and (b) $\beta$ conformations. The panels give the excited state number, associated eigenvalue $(w)$, transition energy $(\omega)$, wavelength $(\lambda)$, and oscillator strength $(f)$. 
(a)

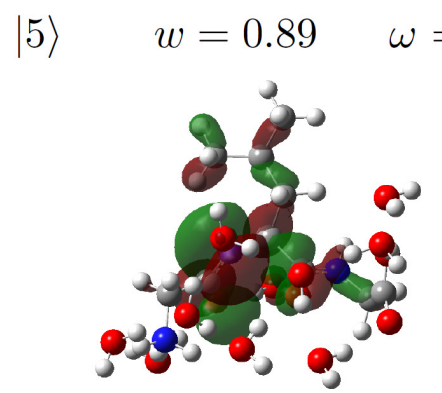

Hole $\lambda=174.90 \mathrm{~nm} \quad f=0.16$

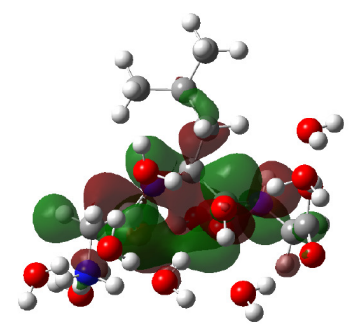

Particle

(b)

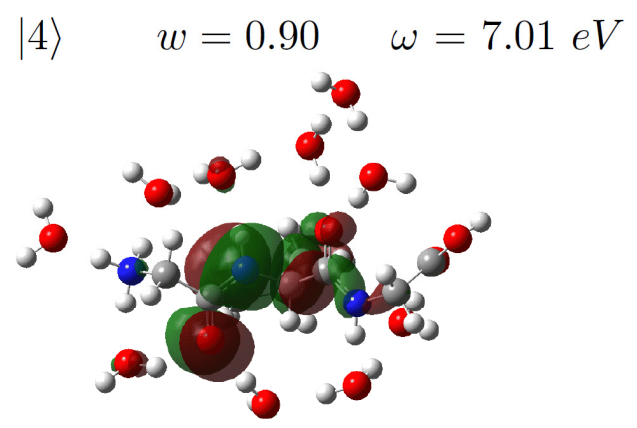

Hole

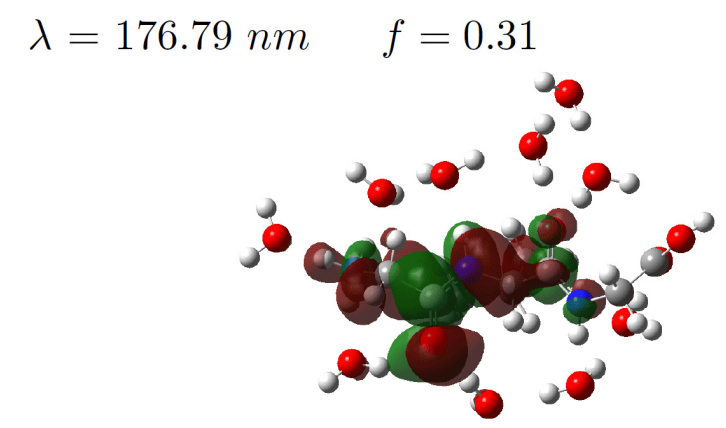

Particle

Figure 8. Natural Transition Orbitals (NTOs) of GLG in the (a) pPII and (b) $\beta$ conformations. The panels give the excited state number, associated eigenvalue $(w)$, transition energy $(\omega)$, wavelength $(\lambda)$, and oscillator strength $(f)$. 
(a)

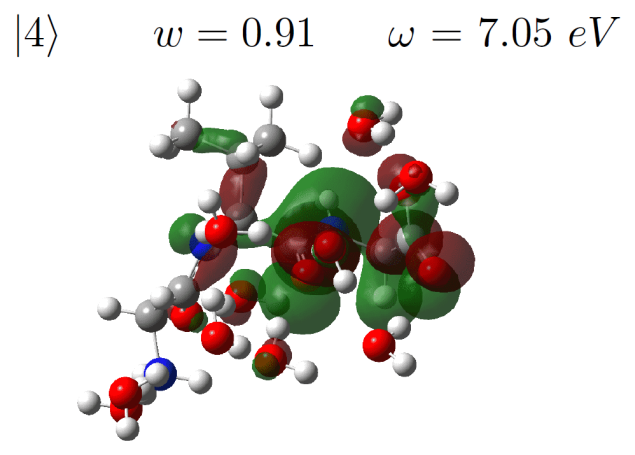

Hole

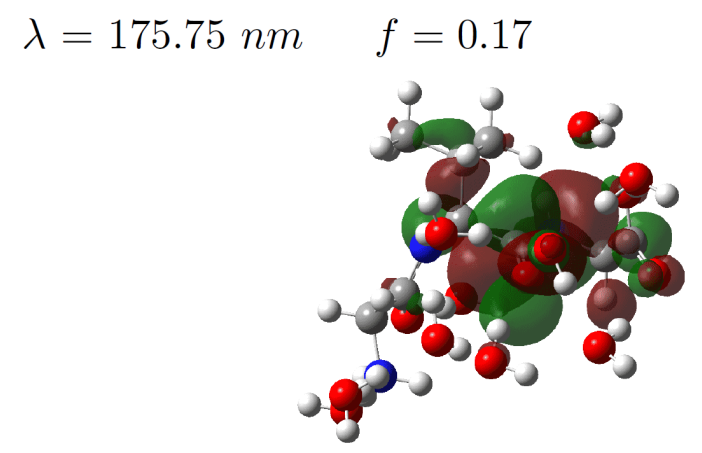

Particle

(b)

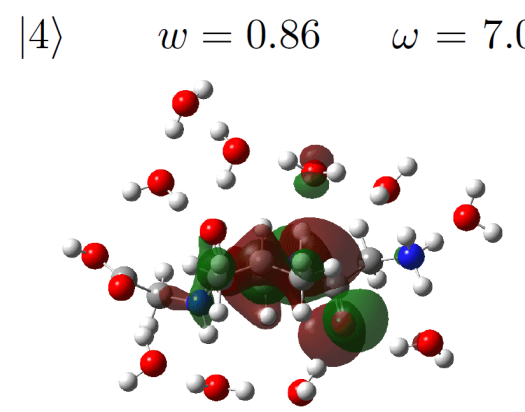

Hole

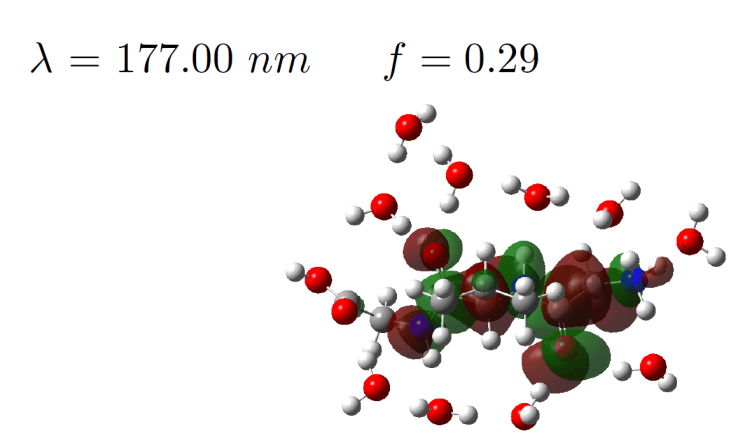

Particle

Figure 9. Natural Transition Orbitals (NTOs) of GVG in the (a) pPII and (b) $\beta$ conformations. The panels give the excited state number, associated eigenvalue $(w)$, transition energy $(\omega)$, wavelength $(\lambda)$, and oscillator strength $(f)$. 
(a)

$|8\rangle$

$$
w=0.97 \quad \omega=7.63 \mathrm{eV}
$$

$\lambda=162.48 \mathrm{~nm} \quad f=0.45$

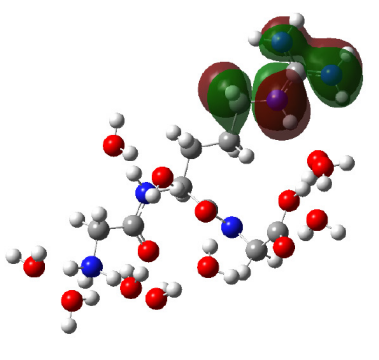

Hole

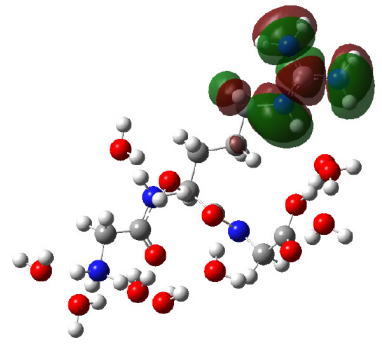

Particle

(b)

$|8\rangle$

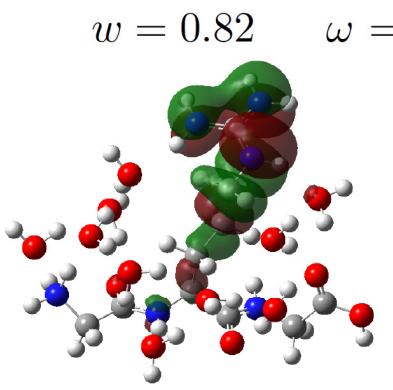

Hole

Figure 10. Natural Transition Orbitals (NTOs) of GRG in the (a) pPII and (b) $\beta$ conformations. The panels give the excited state number, associated eigenvalue $(w)$, transition energy $(\omega)$, wavelength $(\lambda)$, and oscillator strength $(f)$.

For all peptides investigated, the NTOs in explicit water exhibit contributions from water molecules. In the pPII conformations of GIG, GLG, and GVG, the particles of the NTOs responsible for $\mathrm{NV}_{1}$ transition are mostly localized over the carboxylate group. The NTOs of the $\beta$ conformations are more delocalized over the water molecules than the NTOs of the corresponding pPII conformation. This observation is consistent with the previously discussed influence of explicit water on the oscillator strength of both $\mathrm{NV}_{1}$ and $\mathrm{NV}_{2}$.

The $\mathrm{NV}_{1}$ related NTOs of GRG are peculiar in that the dominant transition of the respective pPII conformation is completely localized on the side chain (both hole and particle). The situation is somewhat different in the $\beta$-strand conformation. While transition 8 is still mostly localized on the side chain, transition 19 has some charge transfer character involving a hole localized over the C-terminal peptide, the C-terminal carboxylate group, and water, while the particle is nearly 
entirely on the carboxylate group (Figure 10)

In the $\beta$ conformations of GAG, GIG, GLG, GVG, and GRG, NTOs responsible for the $\mathrm{NV}_{2}$ transition are mostly localized on the water molecules that are around the peptides. For the corresponding pPII conformations of these peptides, the NTOs are mostly on the peptides' main chain, except for GRG in which the NTOs are mostly on the side chain of the peptide.

The $\mathrm{NV}_{2}$ transition peaks in the absorption spectra of the pPII conformation for GAG, GIG, GLG, and GVG redshifts by around $4-5 \mathrm{~nm}$ when using an explicit water model. The corresponding $\mathrm{NV}_{2}$ transition bands of the $\beta$ conformation redshifts by around $7-8 \mathrm{~nm}$. This shift in the $\mathrm{NV}_{2}$ peak suggests again that the absorption spectra of the $\beta$ conformation of the corresponding peptides - GAG, GIG, GLG, and GVG - are affected more by the explicit water model than their pPII counterparts.

Taken together, the results of our NTO analysis suggest that the electronic structure of the ground and excited state of the investigated peptides changes with its conformation and depends on the character of the side chain. The contribution of water in the hydration shell is substantial for the $\mathrm{NV}_{2}$ transition and significant only in the more extended $\beta$-strand conformation. Quite a few of the dominant electronic transitions have a charge-transfer character; some of them involve the side chains. These findings call into question how the electronic system of polypeptides and proteins have been treated thus far. This issue is briefly discussed below.

\section{Comparison with literature}

Over the last 70 years, a multitude of papers has been published on identifying the underlying physics of the $\mathrm{CD}$ and absorption spectra of polypeptides and proteins. In particular, efforts have focused on explaining the spectra of regular secondary structures. Both the oldest and newest attempts are built on the validity of the excitonic coupling model, ${ }^{26,34,35,38,55,56}$ which assumes that the electronic ground state of a polypeptide can be approximately described as a superposition of individual peptide molecular orbitals that are localized on the respective peptide groups. Hence, the respective wavefunction could be written as a product of wavefunctions of individual peptide groups. $\mathrm{NV}_{1}$ excitations, which are generally assigned to a $\mathrm{HOMO} \rightarrow \mathrm{LUMO}$ 
transition, cause a delocalization of electronic states. The coupling between transitions has earlier been described by transition dipole formalism while more recent attempts consider electrostatic interactions between charge distributions. ${ }^{55,56}$ This type of modeling has persisted in the literature because it had a lot of success, particularly in regard to the prediction of the UV-CD spectra of right-handed helical conformations. However, our results suggest that the basic assumptions on which this model is built might be incorrect. First of all, our results suggest that one cannot assign $\mathrm{NV}_{1}$ to a single transition. Second, we found that the character of the transitions and the resulting excited electronic states are side-chain dependent. Some transitions might have a charge transfer character where the electron moves from the side chain to the orbitals that are delocalized over peptide groups. The degree of delocalization in the excited state is conformation and peptide dependent. Moreover, the role of water cannot be neglected for extended $\beta$-strand conformations. Such a direct influence of water has not yet been taken into account in the field.

Another implication of our results deserves to be emphasized. Currently, most of the force fields used for molecular dynamics simulations are built on the assumption that the torsional force with respect to the dihedral angles $\varphi$ and $\psi$ does not depend on the respective side chain. ${ }^{19,21,57-59}$ Only recently have attempts been made to consider the specific influence of side chains on the potential function associated with the backbone dihedral angles. ${ }^{60,61}$ The obtained involvement of side-chain MOs in the highest occupied states of peptides and the partially delocalized character of the electronic ground state argues in favor of side-chain dependent torsional force. The contribution of water orbitals to the electronic ground state, particularly of the $\beta$-strand conformation, suggests the necessity to modify the way how backbone-water interactions are accounted for in molecular dynamic force fields. In this context, the question arises whether force fields with force constants obtained from DFT-calculations on model peptides in the gas phase or even in implicit water can sufficiently describe the energetics and dynamics of peptide/protein backbones. ${ }^{20}$

It is obvious that a more complete picture than that reported in this paper could be gained by TDDFT calculations with a larger number of hydration water molecules. DFT-based geometry optimizations of tripeptides have recently been achieved with up to 30 water molecules. ${ }^{48,62}$ The 
corresponding TDDFT calculations would be very computational expansive. Combined MDTDDFT calculations would only provide an improvement if the hydration shell would become part of the TD-DFT part of the calculation.

\section{Conclusions}

The experimental UV-A spectra of some cationic GxG peptides in water indicate that the two dominant conformations, namely polyproline II and $\beta$-strand, have different absorption spectra. This notion is supported by TDDFT calculations for a selection of aliphatic peptides with a hydration shell of 10 water molecules. Our calculations also account for the UV-CD spectra of these peptides. The computational results strongly suggest that the two canonical $\mathrm{NV}_{1}$ and $\mathrm{NV}_{2}$ bands (the latter is obtainable only with synchrotron radiation) are composed of multiple transitions that result from configurational interactions between numerous transitions. The dispersion of these transitions depends on the backbone conformation and in part on the side chain. Many of the individual transitions have a charge-transfer character. Transitions involving water MOs contribute predominantly to the $\beta$-strand conformation. Our results contradict the basic assumption of the excitonic coupling models that are generally used for the theoretical analysis of $\mathrm{CD}$ and absorption spectra of polypeptides and even proteins.

\section{Acknowledgment}

This project was in part supported by an NSF grant from the National Science Foundation (DMR1707770). A.K. and B.M.W. acknowledge partial support from a UC Riverside Collaborative Seed Grant and the Office of Naval Research (Grant N00014-18-1-2740).

\section{Supporting Information Available}

Additional figures depicting experimental and calculated CD and absorption spectra, optimized geometries, NTOs, and tables listing excitation energies, oscillator, and rotational strengths are 
provided in the Supporting Information.

\section{References}

(1) Hovmoller, S.; Zhou, T.; Ohlson, T. Conformations of Amino Acids in Proteins. Acta Crystallogr. D Biol. Crystallogr. 2002, 58, 768-776.

(2) Ramachandran, G. N.; Ramachandran, C.; Sasisekharan, V. Stereochemistry of Polypeptide Chain Configurations. J. Mol. Biol. 1963, 7, 95-99.

(3) Brant, D. A.; Flory, P. J. J. The Configuration of Random Polypeptide Chains. II. Theory. J. Am. Chem. Soc. 1965, 87, 2791-2800.

(4) Flory, P. J. Statistical Mechanics of Chain Molecules; Wiley \& Sons: New York, 1969.

(5) Garcia-Pietro, F. F.; Galván, I. F.; Aguliar, M. A.; Martin, M. E. Study on the Conformational Equilibrium of the Alanine Dipeptide in Water Solution by Using the Averaged Solvent Electrostatic Potential from Molecular Dynamics Methodology. J. Chem. Phys. 2011, $135,194502$.

(6) Feig, M. Is Alanine Dipeptide a Good Model for Representing the Torsional Preferences of Protein Backbones? J. Chem. Theory Comput. 2008, 4, 1555-1564.

(7) Ho, B. K.; Thomas, A.; Brasseur, R. Revisiting the Ramachandran Plot: Hard-Sphere Repulsion, Electrostatics, and H-Bonding in the Alpha-Helix. Protein Sci. 2003, 12, 2508-2522.

(8) Ho, B. K.; Brasseur, R. The Ramachandran Plots of Glycine and Pre-Proline. BMC Struct. Biol. 2005, 5, 1-14.

(9) Shi, Z.; Woody, R. W.; Kallenbach, N. R. Is Polyproline II a Major Backbone Conformation in Unfolded Proteins? Adv. Protein Chem. 2002, 62, 163-240.

(10) Toal, S. E.; Schweitzer-Stenner, R. Local Order in the Unfolded State: Conformational Biases and Nearest Neighbor Interactions. Biomolecules 2014, 4, 725-773.

(11) Shi, Z.; Chen, K.; Liu, Z.; Ng, A.; Bracken, W. C.; Kallenbach, N. R. Polyproline II Propensities from GGXGG Peptides Reveal an Anticorrelation with $\beta$-Sheet Scales. . Proc. Natl. Acad. Sci. USA 2005, 102, 17964-17968.

(12) Grdadolnik, J.; Mohacek-Grosev, V.; Baldwin, R. L.; Avbelj, F. Populations of the Three Makor Backbone Conformations in 19 Amino Acid Dipeptides. Proc. Natl. Acad. Sci. USA 2011, 108, 1794-1798.

(13) Grdadolnik, J.; Grdadolnik, S. G.; Avbelj, F. Determination of Conformational Preferences of Dipeptides Using Vibrational Spectroscopy. J. Phys. Chem. B 2008, 112, 27122718.

(14) Hagarman, A.; Measey, T. J.; Mathieu, D.; Schwalbe, H.; Schweitzer-Stenner, R. Intrinsic Propensities of Amino Acid Residues in GxG Peptides Inferred from Amide I' Band Profiles and NMR Scalar Coupling Constants. J. Am. Chem. Soc. 2010, 132, 540-551.

(15) Verbaro, D.; Mathieu, D.; Toal, S. E.; Schwalbe, H.; Schweitzer-Stenner, R. Ionized Trilysine: A Model System for Understanding the Nonrandom Structure of Poly-L-Lysine and Lysine-Containing Motifs in Proteins. J. Phys. Chem. B. 2012, 116, 8084-8094.

(16) Toal, S. E.; Kubatova, N.; Richter, C.; Linhard, V.; Schwalbe, H.; Schweitzer-Stenner, R. Randomizing the Unfolded State of Peptides (and Proteins) by Nearest Neighbor Interactions between Unlike Residues. Chem. - A Eur. J. 2015, 21, 5173-5192.

(17) Schweitzer-Stenner, R.; Toal, S. E. Entropy Reduction in Unfolded Peptides (and Proteins) Due to Conformational Preferences of Amino Acid Residues. Phys. Chem. Chem. 
Phys. 2014, 16, 22527-22536.

(18) Parchaňsky, V.; Kapitan, J.; Kaminský, J.; Šebestic, J.; Bouř, P. Ramachandran Plot for Alanine Dipeptide as Determined from Raman Optical Activity. J. Phys. Chem. Lett. 2013, 4, 2763-2678.

(19) Maier, J. A.; Martinez, C.; Kasavajhala, K.; Wickstrom, L.; Hauser, K. E.; Simmerling, C. Ff14SB: Improving the Accuracyof Protein Side Chain and Backbone Parameters from Ff99SB. J. Chem. Theory. Comput. 2015, 11, 3696-3713.

(20) Tian, C.; Kasavajhala, K.; Befon, K. A. A.; Raguette, L.; Huang, H.; Migues, A. N.; Bickel, J.; Wang, Y.; Pincay, J.; Wu, Q.; et al. Ff19SB: Amino-Acid Specific Protein Backbone Parameters Trained against Qunatum Mechanics Energy Surfaces in Solution. J. Chem. Theory Comput. 2019, 16, 528-522.

(21) MacKerell, A. D.; Feig, M.; Brooks, C. L. Extending the Treatment of Backbone Energetics in Protein Force Fields: Limitations of Gar-Phase Quantum Mechanics in Reproducing Protein Conformational Distributions in Molecular Dynamics Simulations. J. Comp. Chem. 2004, 25, 1400-1415.

(22) Toal, S.; Meral, D.; Verbaro, D.; Urbanc, B.; Schweitzer-Stenner, R. PH-Independence of Trialanine and the Effects of Termini Blocking in Short Peptides: A Combined Vibrational, NMR, UVCD, and Molecular Dynamics Study. J. Phys. Chem. B 2013, 117, 3689-3706.

(23) Meral, D.; Toal, S.; Schweitzer-Stenner, R.; Urbanc, B. Water-Centered Interpretation of Intrinsic PPII Propensities of Amino Acid Residues: In Vitro-Driven Molecular Dynamics Study. J. Phys. Chem. B 2015, 119, 13237-13251.

(24) Zhang, S.; Schweitzer-Stenner, R.; Urbanc, B. Do Molecular Dynamics Force Fields Capture Conformational Dynamics of Alanine in Water. J. Chem. Theory Comput. 2020, 16, 510-527.

(25) Woody, R. W. Circular Dichroism of Unordered Polypeptides. Adv. Biophys. Chem. 1992, 2, 37-79.

(26) Woody, R. W. Circular Dichroism Spectrum of Peptides in the Poly(Pro)II Conformation. J. Am. Chem. Soc. 2009, 131, 8234-8245.

(27) Kelly, M. A.; Chellgren, B. W.; Rucker, A. L.; Troutman, J. M.; Fried, M. G.; Miller, A. F.; Creamer, T. P. Host-Guest Study of Left-Handed Polyproline II Helix Formation.

Biochemistry 2001, 40, 14376-14383.

(28) Rucker, A. L.; Creamer, T. P. Polyproline II Helical Structure in Protein Unfolded States: Lysine Peptides Revisited. Protein Sci. 2002, 11, 980-985.

(29) Eker, F.; Griebenow, K.; Schweitzer-Stenner, R. Stable Conformations of Tripeptides in Aqueous Solution Studied by UV Circular Dichroism Spectroscopy. J. Am. Chem. Soc. 2003, $125,8178-8185$.

(30) Toal, S. E.; Verbaro, D. J.; Schweitzer-Stenner, R. Role of Enthalpy-Entropy Compensation Interactions in Determining the Conformational Propensities of Amino Acid Residues in Unfolded Peptides. J. Phys. Chem. B 2014, 118, 1309-1318.

(31) Shi, Z.; Chen, K.; Liu, Z.; Kallenbach, N. R. Conformation of the Backbone in Unfolded Proteins. Chem. Rev. 2006, 106, 1877-1897.

(32) Yang, W. Y.; Larios, E.; Gruebele, M. On the Extended $\beta$-Conformation Propensity of Polypeptides at High Temperature. J. Am. Chem. Soc. 2003, 125, 16220-16227.

(33) Kumar, A.; Schweitzer-Stenner, R.; Wong, B. M. A New Interpretation of the Structure and Solvent Dependence of the Far UV Circular Dichroism Spectrum of Short Oligopeptides. Chem. Comm. 2019, 55, 5701-5704.

(34) Tinoco, I. Theoretical Aspects of Optical Activity .2. Polymers. Adv. Chem. Phys. 1962, 4, 113-160. 
(35) Moffit, M. Optical Rotatory Dispersion of Helical Polymers. J. Chem. Phys. 1956, 25, 467-478.

(36) Sreerama, N.; Woody, R. W. Estimation of Protein Secondary Structure from Circular Dichroism Spectra: Comparison of CONTIN, SELCON, and CDSSTR. Anal. Biochem. 2000, 287, 252-260.

(37) Ham, J. S.; Platt, J. E. Far U.V. Spectra of Peptides. J. Chem. Phys.. 1952, 20, 335-336.

(38) Gratzer, W. B.; Rhodes, W.; Fasman, G. D. Optical Properties of the Poly-L-Proline and Collagen Helices. Biopolymers 1963, 1, 319-330.

(39) Nielsen, E. B.; Schellman, J. A. The Absorption Spectra of Simple Amides and Peptides. J.Phys.Chem. 1967, 1, 2297-2304.

(40) Saidel, L. J.; Goldfarb, A. R.; Waldman, S. The Absorption Spectra of Amino Acids in the Region Two Hundred to Two Hundred and Thirty Millimicrons. J. Biol. Chem. 1952, 197 (1), 285-291.

(41) Dragomir, I. C.; Measey, T. J.; Hagarman, A. M.; Schweitzer-Stenner, R. EnvironmentControlled Interchromophore Charge Transfer Transitions in Dipeptides Probed by UV Absorption and Electronic Circular Dichroism Spectroscopy. J. Phys. Chem. B 2006, 110, $13235-13241$.

(42) Chen, X. G.; Asher, S. A.; Schweitzer-Stenner, R.; Mirkin, N. G.; Krimm, S. UV Raman Determination of the $\Pi \pi^{*}$ Excited State Geometry of N-Methylacetamide: Vibrational Enhancement Pattern. J. Am. Chem. Soc. 1995, 117, 2884-2895.

(43) Frisch, M. J.; Trucks, G. W.; Schlegel, H. B.; Scuseria, G. E.; Robb, M. A.; Cheeseman, J. R.; Scalmani, G.; Barone, V.; Petersson, G. A.; Nakatsuji, H.; et al. Gaussian 09 Revision E.01. 2016 .

(44) Ilawe, N. V; Raeber, A. E.; Schweitzer-Stenner, R.; Toal, S. E.; Wong, B. M. Assessing Backbone Solvation Effects in the Conformational Propensities of Amino Acid Residues in Unfolded Peptides. Phys. Chem. Chem. Phys. 2015, 17, 24917-24924.

(45) Kaminský, J.; Kubelka, J.; Bouř, P. Theoretical Modeling of Peptide $\alpha$-Helical Circular Dichroism in Aqueous Solution. J. Phys. Chem. A 2011, 115, 1734-1742.

(46) Šebek, J.; Kejík, Z.; Bouř, P. Geometry and Solvent Dependence of the Electronic Spectra of the Amide Group and Consequences for Peptide Circular Dichroism. J. Phys. Chem. A 2006, 110, 4702-4711.

(47) Šebek, J.; Gyurcsik, B.; Šebestík, J.; Kejík, Z.; Bednárová, L.; Bouř, P. Interpretation of Synchrotron Radiation Circular Dichroism Spectra of Anionic, Cationic, and Zwitterionic Dialanine Forms. J. Phys. Chem. A 2007, 111, 2750-2760.

(48) Lanza, G.; Chiacchio, M. A. Ab Initio MP2 and Density Functional Theory Computational Study of AcAlaNH2 Peptide Hydration: A Bottom-Up Approach. ChemPhysChem 2014, 15, 2785-2793.

(49) Neto, A. P. V; Machado, D. F. S.; Lopes, T. O.; Camargo, A. J.; de Oliveira, H. C. B. Explicit Aqueous Solvation Treatment of Epinephrine from Car-Parrinello Molecular Dynamics: Effect of Hydrogen Bonding on the Electronic Absorption Spectrum. J. Phys. Chem. B 2018, $122,8439-8450$.

(50) Cupane, A.; Leone, M.; Vitrano, E.; Cordone, L. Low Temperature Optical Absorption Spectroscopy: An Approach to the Study of Stereodynamic Properties of Hemeproteins. Eur. Biophys. J. 1995, 23, 385-398.

(51) Cupane, A.; Leone, M.; Unger, E.; Lemke, C.; Beck, M.; Dreybrodt, W.; SchweitzerStenner, R. Dynamics of Various Metal-Octaethylporphyrins in Solution Studied by Resonance Raman and Low-Temperature Optical Absorption Spectroscopies. Role of the Central Metal. $J$. Phys. Chem. B 1998, 102, 6612-6620. 
(52) Hagarman, A.; Measey, T. J.; Mathieu, D.; Schwalbe, H.; Schweitzer-Stenner, R. Intrinsic Propensities of Amino Acid Residues in GxG Peptides Inferred from Amide I' Band Profiles and NMR Scalar Coupling Constants. J. Am. Chem. Soc. 2010, 132, 540-551.

(53) Schweitzer-Stenner, R.; Hagarman, A.; Toal, S.; Mathieu, D.; Schwalbe, H. Disorder and Order in Unfolded and Disordered Peptides and Proteins: A View Derived from Tripeptide Conformational Analysis. I. Tripeptides with Long and Predominantly Hydrophobic Side Chains. Proteins Struct. Funct. Bioinforma. 2013, 81, 955-967.

(54) Rybka, K.; Toal, S. E.; Verbaro, D. J.; Mathieu, D.; Schwalbe, H.; Schweitzer-Stenner, R. Disorder and Order in Unfolded and Disordered Peptides and Proteins: A View Derived from Tripeptide Conformational Analysis. II. Tripeptides with Short Side Chains Populating Asx and $\beta$-Type like Turn Conformations. Proteins Struct. Funct. Bioinforma. 2013, 81, 968-983.

(55) Jiang, J.; Abramavicius, D.; Bulheller, B. M.; Hirst, J. D.; Mukamel, S. Ultraviolet Spectroscopy of Protein Backbone Transitions in Aqueous Solution: Combined QM and MM Simulations. J. Phys. Chem. B 2010, 114, 8270-8277.

(56) Hirst, J. D.; Colella, K.; Gilbert, A. T. B. Electronic Circular Dichroism of Proteins from First-Principles Calculations. J. Phys. Chem. B 2003, 107, 11813-11819.

(57) Robertson, M. J.; Tirado-Rives J.; Jorgensen, W. L. Improved Peptide and Torsionla Energetics with the OPLS-AA Force Field. J. Chem. Theory Comput. 2015, 11, 3499-3509.

(58) Best, R. B.; Hummer, G. Optimized Molecular Dynamics Force Fields Applied to the Helix-Coil Transition of Polypeptides. J. Phys. Chem. B 2009, 113, 9004-9015.

(59) MacKerell, A. D.; Bashford, D.; Bellot, M.; Dunbrack, R. L.; Evanseck, J. D.; Field, M. J.; Fischer, S.; Gao, J.; Guo, H.; Ha, S.; et al. All Atom Empirical Potential for Molecular Modeling and Dynamic Studies of Proteins. J. Phys. Chem. B 1998, 102, 3586-3616.

(60) Zhou, C.-Y.; Jiang, F.; Wu, Y.-D. Residue-Specific Force Field Based on Protein Coil Library. RSFF2: Modification of Amber Ff99SB. J. Phys. Chem. B 2014, 119, 1035-1047.

(61) Li, S.; Elcock, A. H. Residue-Specific Force Field (RSFF2) Improves the Modeling of Conformational Behavior of Peptides and Proteins. J. Phys. Chem. Lett. 2015, 6, 2127-2133.

(62) Lanza, G.; Chiacchio, M. A. Comprehensive and Accurate Ab Initio Energy Surface of Simple Alanine Peptides. ChemPhysChem 2013, 14, 3284-3293. 


\section{Spectra via TDDFT}
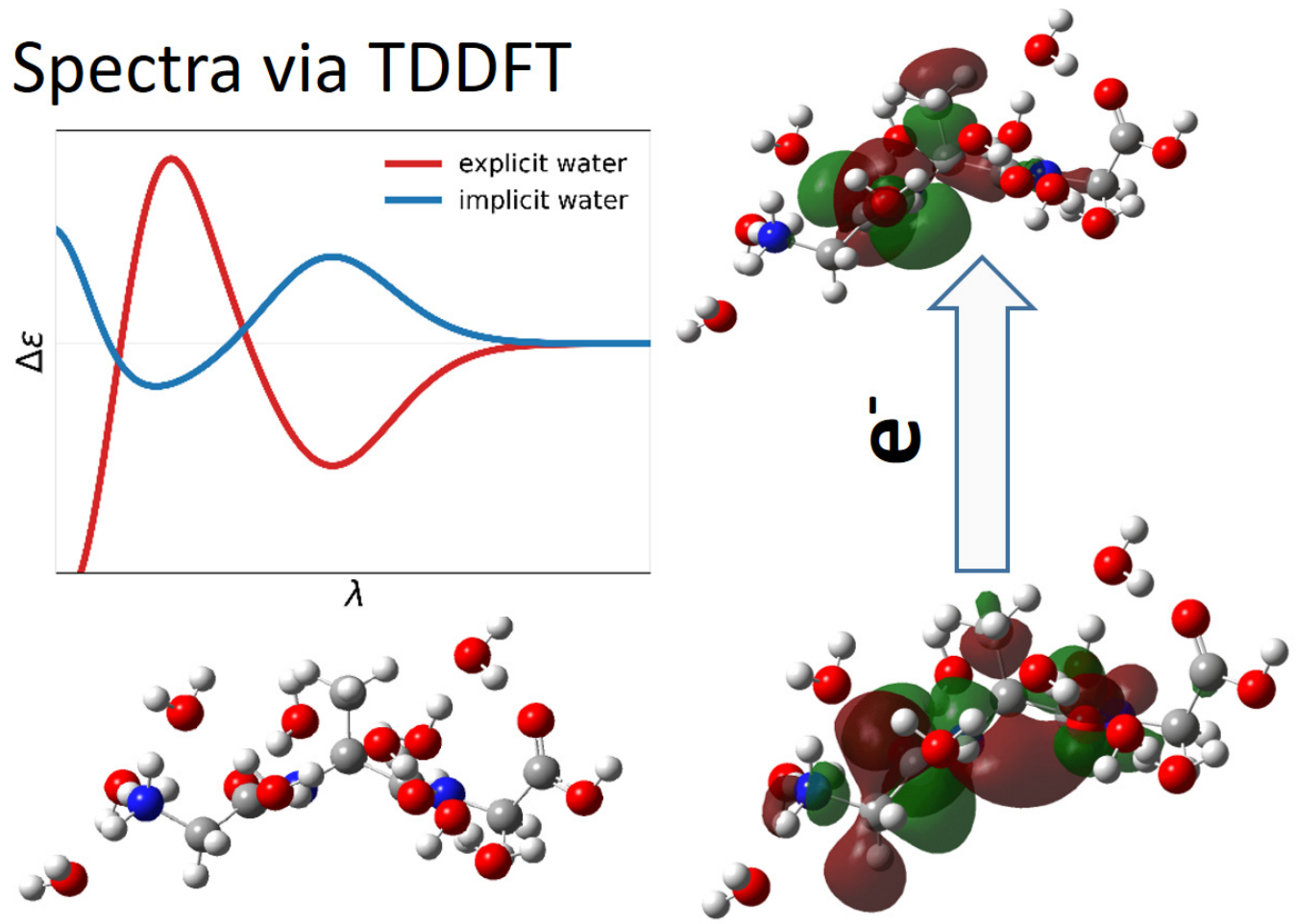

TOC Graphics 